\title{
The Spanish Society of Drug Addiction
}

\begin{tabular}{|l|l|l|}
\hline L.S & Luis & San \\
\hline
\end{tabular}

Hospital del Mar, Servei de Psipuiatria i Toxicomanies, Barcelona, España

Dr. Luis San, Hospital del Mar, Servei de Psipuiatria i Toxicomanies, Passeig Maritim, 25-39, E-08003 Barcelona (Spain) In the last few years, a renewed public, governmental and professional interest has appeared towards the recognition, assessment and treatment of substance abuse disorders. The reason is quite obvious: addictive disorders are associated with a high morbidity and mortality and contribute to the genesis of behavioural disturbances.

Despite these important personal and social costs, it has not until very recently been that health professionals and, in particular psychiatrists, have become increasingly aware of the growing need for treatment, research and teaching in the area of drug addiction. However, in view of the profound effects of these disorders on cognitive development, personality traits and social functioning, the medical profession is now more sensitive to progress in the knowledge of drug addiction.

The Spanish Society of Drug Addiction (Sociedad Espanola de Toxicomanfas, SET) was founded in December 1988. In addition to the considerations mentioned above, there were other reasons for the establishment of SET including, firstly, the dramatic increase in the number of drug users (alcohol and other illegal drugs) and drug consumption-derived health care problems, and, secondly, the need to have a framework available for the scientific exchange of experiences among professionals involved in the area of drug abuse research. The presence of SET supplements the already existing scientific associations in the field of psychiatry and mental health.

Since the foundation of SET, different Spanish public and private institutions, such as Plan Nacional sobre Dro-gas, FIS, CAYCIT, CIRIT or CITRAN, have actively cooperated in the aims and establishment of the Society, in particular, by supporting the development of research in neurosciencies and clinical studies.

The main purpose of the Society is to promote all kinds of scientific activities and to encourage the general progress of knowledge in this rapidly changing field. SET therefore supports, organizes, and participates in symposia, meetings, programs or activities considered to be of interest for the achievement of the aims. The Society also provides its members with the necessary information to obtain grants and financial support from either public or private institutions.

It is a nationwide scientific society, and most of its members are physicians, psychiatrists and psychologists involved in different research activities in the field of drug addiction, such as basic and experimental investigations, clinical trials, epidemiological surveys, and toxicological studies. The majority of these research groups are linked to universities, research institutions or teaching hospitals of our national health care system.

The Board of Trustees is composed by one chairman (L. San), one vice-chairman (M. Gutierrez), one secretary (J. Garzon), one treasurer (J. Sisquellas) and five committee members (M. Casas, 
G. Cervera, J. Garcia-Sevilla, C. Guerri, C. Lora-Tamayo). Candidates are elected by the General Ordinary Assembly for a period of two renewable years. The General Ordinary Assembly takes place every 2 years concomitantly to the celebration of the congress of the Society.

This congress constitutes one of SET main activities. So far, four congresses have been organized and held in Barcelona (1988), Vitoria (1989), Madrid (1991), and Valencia (1993), respectively. The next meeting will be held in Santiago de Compostela in 1995. The Board of Trustees nominates a Scientific Committee which is responsible for selecting the topics and inviting speakers to participate in the meeting as well as reviewing communications and posters in order to guarantee the scientific standards of all presentations. Main topics of the last congresses were related to transduction of cell receptors in tolerance and dependence developments; recent advances in the mechanism of action of potentially addictive substances; pharmacotherapy of alcohol dependence; epidemiology, psychopathology and risk behaviours in drug addiction; molecular mechanisms involved in drug dependence; new strategies in the treatment of drug abuse; treatment of nicotine dependence; abuse liability of ben-zodiazepines; addiction to psychostimulants; assessment of health care resources; professional training in drug addiction; drug use in the work place; and treatment with opioid antagonists.

To become a member of the Sociedad Espanola de Toxicomanias, which currently has 220 members, it is necessary to fill in an application form with the support of two members, to be accepted by the Board of Trustees and to confirm the acceptance of the candidate by the Ordinary Assembly. Scientists of foreign societies in the field of drug abuse are welcome to become SET members.

Contact: Sociedad Espanola de Toxicomanias (SET),

Head office: C/ Numancia 207, bajos, E-08017 Barcelona (Spain), Tel. 343-2806102; Fax 343-2802756.

65 